\title{
Desenvolvimento e avaliação de jogo eletrônico interativo para o processo de aprendizagem do tratamento de crianças e adolescentes com distrofia muscular de Duchenne*
}

\section{Development and evaluation of an interactive electronic game for the learning process of the treatment of children and adolescents with Duchenne muscular dystrophy}

\author{
Adriana Nathalie Klein ${ }^{1}$, Michele Emy Hukuda², Ana Grasielle Dionísio Corrêa ${ }^{3}$, \\ Irene Karaguilla Ficheman ${ }^{4}$, Roseli Deus Lopes ${ }^{5}$
}

http://dx.doi.org/10.11606/issn.2238-6149.v26i2p281-287

\begin{abstract}
Klein NA, Hukuda ME, Corrêa AGD, Ficheman IK, Lopes RD. Desenvolvimento e avaliação de jogo eletrônico interativo para o processo de aprendizagem do tratamento de crianças e adolescentes com distrofia muscular de Duchenne. Rev Ter Ocup Univ São Paulo. 2015 maio-ago.;26(2):281-7.
\end{abstract}

RESUMO: O trabalho visa apresentar o processo de desenvolvimento e avaliação de um jogo eletrônico interativo criado para favorecer a construção de conhecimento sobre o programa de reabilitação de crianças e adolescentes com Distrofia Muscular de Duchenne (DMD). A coleta de dados do conteúdo do jogo foi feita por brainstorms com equipe multidisciplinar de profissionais da saúde (fisioterapeutas e terapeutas ocupacionais) e pacientes com DMD. Os dados foram analisados e separados em três categorias: genética, fisiopatologia e programas de alongamento. Transformou-se essas informações em storyboard e realizou-se a programação e execução do jogo feitas por uma equipe de engenheiros. Por fim, o jogo eletrônico foi testado por pacientes com DMD por meio de um questionário pré-pós-jogo.

\begin{abstract}
Os resultados demonstraram aumento no repertório relacionado à reabilitação pré-adquirido e pós-jogo. O jogo eletrônico interativo DuchsVille foi criado, avaliado e pode ser utilizado como uma ferramenta de tecnologia de informação aplicável para favorecer processos de ensino e aprendizagem sobre aspectos de reabilitação na DMD, aproximando o diálogo da lógica técnico-científica dos profissionais da saúde da lógica do senso comum da população.
\end{abstract}

DESCRITORES: Distrofia muscular de Duchenne/reabilitação; Educação em saúde; Fisioterapia; Terapia ocupacional; Jogos experimentais.

Klein NA, Hukuda ME, Corrêa AGD, Ficheman IK, Lopes RD. Development and evaluation of an interactive electronic game for the learning process of the treatment of children and adolescents with Duchenne muscular dystrophy. Rev Ter Ocup Univ São Paulo. 2015 May-Aug.;26(2):281-7.

\footnotetext{
* Trabalho apresentado no TOM (Tikkun Olam Make-A-Thon), São Paulo, Universidade de São Paulo, 28-30 nov. 2014. Referência ao trabalho como parte integrante de dissertação, tese ou projeto:

Klein AN. Araújo. Criação de um questionário de conhecimento em Distrofia Muscular de Duchenne (Muscular Dystrophy Knowledge Questionnaire)-DDMKQ. Rev Bras Educ Saúde. 2014;4:7-18.

Corrêa AGD, Klein NA, Savioni CC, Ficheman IK, Lopes RD. Desenvolvimento e avaliação do Jogo DuchsVille para apoiar o processo de aprendizagem nutricional:estudo de caso com adolescentes com Distrofia Muscular de Duchenne. RENOTE. Rev Novas Tecnol Educ. 2013;11:1-10.

1. Terapeuta ocupacional formada pela UFSCar, doutoranda do programa de pós-graduação de Engenharia elétrica da Escola Politécnica da Universidade de São Paulo.

2. Fisioterapeuta formada pela Universidade Metodista de São Paulo, Doutora em Ciências da reabilitação pela Faculdade de medicina da USP.

3. Professora Assistente I de Ciência da Computação e Sistemas de informação da Universidade Presbiteriana Mackenzie e Pesquisadora do laboratório de Sistemas Integráveis da Escola Politécnica da Universidade de São Paulo.

4. Pesquisadora do Laboratório de Sistemas Integráveis da Escola Politécnica da Universidade de São Paulo.

5. Professora Associada 3 do Departamento de Engenharia de Sistemas Eletrônicos da Escola Politécnica da Universidade de São Paulo. Endereço para correspondência: adiklein@ig.com.br.
} 
ABSTRACT: This paper aims to present the development and evaluation process of an interactive video game created to promote the construction of knowledge about the rehabilitation program of children and adolescents with Duchenne Muscular Dystrophy (DMD). The game content data collection was done by brainstorming with a multidisciplinary team of health professionals (physiotherapists and occupational therapists) and patients with DMD. Data were analyzed and separated into three categories: genetic, pathophysiology and stretching programs. This information was transformed into a storyboard and a team of engineers made the programming and implementation of the game. Lastly, the electronic game was tested by DMD patients by

\section{INTRODUÇÃO}

A Distrofia Muscular de Duchenne (DMD) é uma doença hereditária caracterizada pela progressiva irreversível degeneração da musculatura esquelética, resultando em fraqueza muscular generalizada, em consequência da mutação do cromossomo Xp21, fator que limita a produção da distrofina, proteína essencial para a função do músculo estriado ${ }^{1,2}$. Trata-se de uma patologia de herança recessiva que afeta o gênero masculino com uma incidência de 1 em cada 3.500 nascidos vivos².

Os primeiros sintomas da DMD iniciam-se a partir dos 3 anos de idade, quando o comprometimento simétrico da musculatura da cintura pélvica reflete nas dificuldades que a criança apresenta em subir escadas, pular e correr ${ }^{3,4}$. Entre 9 e 12 anos geralmente ocorre a perda da capacidade de deambulação, e a pessoa passa a se locomover com cadeira de rodas ${ }^{3}$. A partir desta fase, acontece o maior comprometimento da capacidade respiratória ${ }^{4}$.

Com todo esse quadro clínico descrito, sabese que a pessoa com DMD irá progressivamente perder a capacidade para realizar, de forma independente, suas atividades de vida diária (autocuidados, alimentação, locomoção, entre outros) e de vida prática (atividades na comunidade $)^{2,4}$. Os familiares desses pacientes tornamse responsáveis por inúmeras tarefas cotidianas, como administrar os medicamentos prescritos, realizar os exercícios respiratórios e alongamentos musculares, colocar e retirar as órteses para membros inferiores e superiores, posicionar corretamente na cadeira de rodas, realizar as transferências necessárias, ligar e monitorar os aparelhos de assistência ventilatória ${ }^{5}$. Além disso, devem acompanhar a rotina de atendimento em centros de reabilitação, consultas e exames periódicos ${ }^{6}$.

A abordagem de uma doença complexa, como a DMD, requer comunicação efetiva para a transmissão a pre-post-game questionnaire. The results showed an increase in the repertoire related to rehabilitation after they played the game. The interactive electronic game DuchsVille was created, evaluated and was able to be used as a tool of information technology applicable to favor processes of teaching and learning about rehabilitation aspects of DMD, bringing the technical and scientific logic of health care providers closer to the logic of common sense of the population.

KEYWORDS: Muscular dystrophy, Duchenne/rehabilitation; Health education; Physical therapy specialty; Occupational therapy; Games, experimental.

de informação e cuidados de saúde. Como é uma doença crônica de caráter progressivo e as primeiras manifestações clínicas estão presentes a partir dos três anos de idade, fica difícil a orientação focada no paciente ainda criança. Portanto, nos primeiros sinais e sintomas e no fechamento do diagnóstico, todas as ações de orientação, incluindo o estímulo ao aconselhamento genético, são direcionadas aos familiares da criança com $\mathrm{DMD}^{3,6}$.

A partir dos 6 anos de idade torna-se interessante pensar em estratégias de ensino sobre a doença DMD, para atingir maior compreensão e aderência da criança em sua rotina de tratamento, porém alguns estudos sugerem que cerca de $30 \%$ a $50 \%$ das pessoas com DMD possuem algum grau de comprometimento cognitivo de causa ainda não identificada ou conclusiva. O motivo é que a proteína distrofina também é encontrada no cérebro e a deficiência desta está associada a déficits intelectuais ${ }^{7,8}$, fator que pode prejudicar ainda mais os processos educativos em saúde.

Apesar de não haver dados representativos, sabe-se que há muitas pessoas com DMD ainda sem assistência, e outras que são atendidas apenas em sistema ambulatorial pela equipe de saúde. O tempo de atendimento pode não ser suficiente para que as informações sobre a doença e seu manejo sejam transmitidas por toda equipe interdisciplinar envolvida ${ }^{9,10}$. Dessa forma, a criação de um jogo eletrônico interativo propõe facilitar o processo de ensino-aprendizagem de informações sobre reabilitação que devem ser adotadas no cotidiano. Além do fato de o jogo estar disponível na web, a ferramenta interativa pode atingir um maior número de pacientes com DMD, seus cuidadores e familiares de todo o território brasileiro.

O jogo eletrônico foi financiado pelo edital de apoio a projetos em fase final de desenvolvimento tecnológico de ajudas técnicas do Centro Nacional de TecnologiaAssistivaEstudos e Pesquisas para elaboração de Proposta de Implantação - CNPq processo n. 48.6257/2007-0. 


\section{OBJETIVOS}

O objetivo geral consiste em apresentar o processo de desenvolvimento e avaliação de um jogo eletrônico interativo criado para favorecer a construção de conhecimento sobre o programa de reabilitação de crianças e adolescentes com DMD.

Os objetivos específicos são: identificar o conhecimento de profissionais e de pacientes sobre aspectos relacionados à reabilitação em DMD; abordar a transdisciplinariedade; permitir a participação ativa dos pacientes na construção e revisão do jogo.

\section{MÉTODO}

Para desenvolvimento desta pesquisa foram obedecidos os aspectos éticos da resolução 196/96 do Conselho Nacional de Saúde (CNS), que regulamenta a pesquisa envolvendo seres humanos, respaldada pelo Comitê de Ética em Pesquisa da Universidade Federal de São Paulo, sob o número 0488/10. Todos os participantes assinaram o TCLE.

Trata-se de um estudo prospectivo exploratório, que teve duração de dezembro de 2010 a dezembro de 2102, sendo o jogo DuchesVille disponibilizado na web em janeiro de 2013.

\section{Coleta de dados para criação do conteúdo educativo}

Participaram da coleta de dados sete profissionais de reabilitação (fisioterapeutas e terapeutas ocupacionais), com formação mínima de especialista ou mestre da Associação Brasileira de Distrofia Muscular (ABDIM) e com pelo menos dois anos de experiência no atendimento de pacientes com DMD, e dez pacientes com DMD da ABDIM, deambuladores e cadeirantes, alfabetizados (ensino fundamental ao ensino superior) e com idade entre 11 e 29 anos. Realizou-se um brainstorm, ferramenta para geração de novas ideias, conceitos e soluções para resolver problemas específicos e estimular o pensamento criativo, sobre qual conteúdo relacionado à reabilitação do paciente com DMD seria importante estar contido no jogo educativo. Todos os discursos foram anotados e transcritos em tabelas.

\section{Análise de dados para criação do conteúdo educativo}

Os dados coletados foram separados em quatro categorias, a saber, "genética", "fisiopatologia", "programas de alongamento" e "uso de cadeira de rodas e órteses", devido à frequência dessas citações nos discursos dos profissionais e pacientes entrevistados.

\section{Execução do jogo eletrônico}

Nessa etapa o conteúdo categorizado foi transformado em storyboard (desenhos) por uma equipe composta de duas engenheiras, uma estudante de Design e uma terapeuta ocupacional. As ilustrações foram feitas com uso de mesa digitalizadora Intuos PTK-440. Para ilustração e design foram usados os softwares Adobe Photoshop e Adobe Illustrator, que possibilitam exportar arquivos no formato suportado pelo Adobe Flash (TIF). Alguns vetores foram exportados em PNG, formato escolhido por suportar transparência.

O jogo foi executado com a ferramenta Adobe Flash CS5, que possibilita o acesso pela internet por meio de um navegador web com FlashPlayer (versão $10 \mathrm{ou}$ superior) instalado. A programação do jogo (linkagem das telas, animações e interações com o usuário) foi criada na linguagem de programação ActionScript 3.0 (AS3), suportada pelo Adobe Flash CS5.

\section{Método de avaliação do jogo eletrônico com pacientes com DMD}

Depois de desenvolvido e executado, o jogo foi testado diretamente com dez pacientes com DMD durante uma semana, sendo cinco usuários de cadeira de rodas e cinco deambuladores com idades entre 11 e 29 anos, alfabetizados ou não, e que não fizeram parte do braimstorm inicial. Aplicou-se um questionário quali-quantitativo ilustrado, com objetivo de registrar o conhecimento prévio do usuário sobre aspectos relacionados à reabilitação. Em seguida, o usuário foi convidado a jogar e, ao finalizar, o questionário foi reaplicado para avaliar se as informações foram assimiladas após o jogo. Algumas outras informações também foram coletadas por meio da escala de Likert para avaliar questões relacionadas à estética (contraste das cores, desenhos dos cenários, personagem principal), áudios (vocalização, trilhas sonoras e efeitos sonoros) e usabilidade (facilidade de interação, simplicidade das telas e memorização). Por fim, os jogadores foram convidados a opinar livremente sobre as percepções do jogo.

\section{Método de avaliação do jogo eletrônico com profissionais}

A avaliação de usabililidade do jogo de reabilitação foi submetida a cinco profissionais da área de engenharia e dois profissionais na área de reabilitação (fisioterapeuta 
e terapeuta ocupacional), por meio de um questionário semiestruturado baseado nas heurísticas de Nielsen ${ }^{11}$, incluindo a visibilidade do estado do jogo, o diálogo simples e natural, o controle e a liberdade do usuário, minimizar a carga de memorização para o usuário, a consistência e a aderência a padrões, a estética e o projeto minimalista, as mensagens de erro precisas e construtivas, a prevenção de erros, a ajuda e a documentação.

\section{RESULTADOS}

Desenvolvimento do jogo eletrônico "Duchsville Reabilitação"

O jogo eletrônico foi desenvolvido com os dados coletados e analisados dos quatro fisioterapeutas e três terapeutas ocupacionais, com tempo médio de 5,4 anos de experiência no atendimento de pacientes com DMD, e dez pacientes com DMD, média de idade de 17,1 anos (11-29 anos). O personagem principal foi "caracterizado" como um adolescente do gênero masculino que pode ser deambulador ou cadeirante, dependendo da escolha do usuário; o personagem do terapeuta é responsável por explicações sobre a fisiopatologia da DMD; a ilustração do cachorro interage complementando algumas informações em diversas fases do jogo.

As animações iniciais do jogo explicam a origem, a causa e sobre o principal sintoma clínico da DMD (Figuras 1 e 2). Na etapa seguinte, o usuário deve escolher se quer iniciar o jogo com o personagem deambulador ou o usuário de cadeira de rodas.

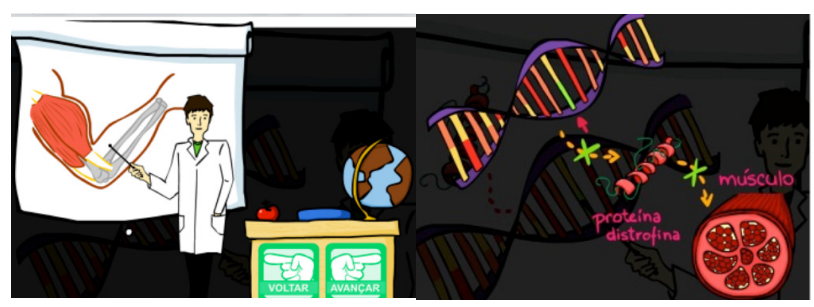

Figuras 1 e 2 - Explicação sobre a genética, a fisiopatologia e o sintoma da DMD

Dependendo da escolha supracitada do usuário, no primeiro jogo ele deve "clicar" nas principais regiões do corpo que precisam ser prioritariamente alongadas com informações visuais e sonoras, e, caso acerte todas as que devem receber a intervenção, passa para o segundo jogo.

O segundo jogo é o das órteses, no qual o usuário é instruído sobre a importância desses dispositivos reabilitadores, e o desafio é encaixá-los de acordo com a necessidade do personagem, cadeirante (Figura 3) ou deambulador (Figura 4), para passar para a terceira etapa.

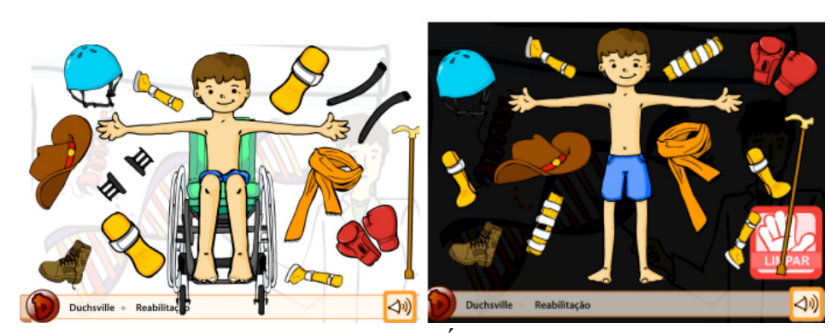

Figuras 3 e 4 - Cenários do jogo Órteses

No terceiro e último, o usuário circula por um cenário de uma casa com seis cômodos e deve achar os equipamentos de reabilitação escondidos (Figura 5). Esse jogo reforça o aprendizado sobre o uso e o cuidado com as órteses que fazem parte da sua rotina diária.

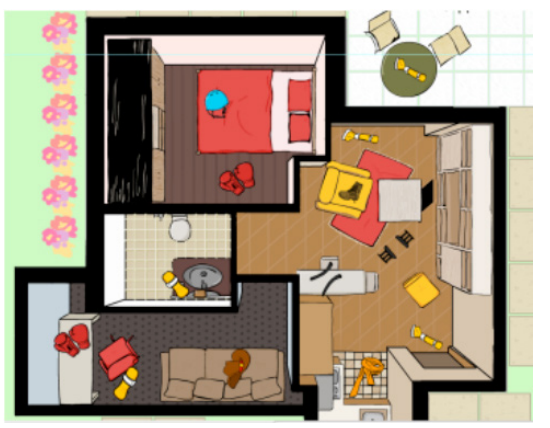

Figura 5 - Cenário do jogo Casa bagunçada

Ao finalizar todas as etapas, o usuário recebe um feedback positivo em relação ao alongamento nas regiões corretas, ao encaixe das órteses de maneira adequada e ao cuidado que deve ter com seus equipamentos de reabilitação. Na tela final, o jogador poderá deixar seu nome registrado e interagir com outros usuários.

\section{Resultados do impacto do processo de aprendizagem dos pacientes com DMD - Teste pré e pós-jogo e percepções qualitativas}

A análise comparativa dos resultados pré e pós-jogo relacionados ao tema "alongamento" demonstrou que os pacientes usuários de cadeira de rodas apresentaram um aumento do repertório das regiões em $62 \%$, e $38 \%$ mantiveram o mesmo repertório. Nos pacientes deambuladores avaliados 
para esse mesmo tema, $50 \%$ manifestaram algum acréscimo no conteúdo prévio, $33 \%$ indicaram igual repertório pré e pós-jogo e em $17 \%$ não foi possível classificar.

A análise dos resultados do tema "órteses" demonstrou que $50 \%$ dos participantes usuários de cadeira de rodas apresentaram um aumento de repertório pós-jogo e $50 \%$ não obtiveram diferenças. Nos pacientes deambuladores avaliados, 33\% tiveram aumento de repertório pós-jogo, $50 \%$ manifestaram as mesmas respostas pré e pós-jogo e em $17 \%$ não foi possível classificar.

A opinião dos jogadores em relação à estética do jogo foi: $29 \%$ classificaram como excelente; $33 \%$, muito bom; $26 \%$, bom; $9 \%$, regular; e $2 \%$, ruim. Quanto à avaliação do áudio do jogo, $36 \%$ classificaram como excelente; $26 \%$, muito bom; $33 \%$, bom; e $5 \%$, regular; sobre o item usabilidade do jogo, $40 \%$ classificaram como excelente; $18 \%$, muito bom; $32 \%$, bom; $8 \%$, regular; $2 \%$, ruim; e $2 \%$, péssimo.

Por fim, a análise dos dez discursos qualitativos permitiu a seleção das expressões-chave, e destas extraiuse quatro ideias centrais que tiveram as frequências contabilizadas: $50 \%$ das opiniões foram afirmações positivas (elogios gerais) relacionadas ao jogo, $50 \%$ das respostas foram opiniões relacionadas a quanto o jogo favorece processos de aprendizagem sobre DMD, $20 \%$ opiniões ligadas ao aumento de repertório do próprio participante e $30 \%$ das opiniões foram sugestões para melhoria do jogo em diversos aspectos.

\section{Resultados da avaliação dos peritos (ajustes realizados e ferramenta educativa)}

A análise das opiniões dos peritos indicou diversos problemas nos aspectos de tecnologia e usabilidade, como erros na velocidade das animações, falta de opção para repetir as sequências, dificuldade para acessar partes do jogo ou sair dele, e mau funcionamento dos botões de acesso.

As informações supracitadas foram adicionadas às sugestões qualitativas dos pacientes e a equipe pôs em prática as correções e as adequações ao software. O jogo foi hospedado no site da Abdim (www.abdim.org.br) e está disponível para o público em geral.

\section{DISCUSSÃO}

Com os avanços significativos na área da tecnologia de educação, novas formas de processos de ensino e aprendizagem tornaram-se possíveis ${ }^{12}$. A interatividade da tecnologia, como um transmissor de informações, faz que o usuário, com o uso do computador/tablet/smartphone, construa seu próprio conhecimento ${ }^{13}$.
Nesse mesmo caminho, diversos estudos tentam provar a efetividade de programas educativos relacionados à saúde via internet, mas um dos desafios é a fonte segura das informações ${ }^{14}$. Alguns conseguiram mostrar vantagens nesse tipo de técnica educativa, como no caso da artrite reumatoide ${ }^{15}$, da fibromialgia ${ }^{14,16} \mathrm{e}$ da esclerose lateral amiotrófica ${ }^{17}$.

O uso de jogos eletrônicos via web como mediador do aprendizado de crianças e adolescentes tem sido um assunto emergente como estratégia didática para processos de educação em saúde ${ }^{18}$. A aplicabilidade dos jogos tem natureza lúdica e informativa, propicia a interação humana ${ }^{19} \mathrm{e}$ faz do participante um agente ativo no processo de aprendizagem ${ }^{20}$.

Ensinar crianças e adolescentes sobre sua doença é um grande desafio. Por se tratar de doenças de caráter progressivo, a abordagem deve ser bem cuidadosa ${ }^{21}$. Em diversos países desenvolvidos, existem acampamentos de férias para crianças com distrofia muscular ${ }^{22}$, e no Japão alguns hospitais especializados são moradias coletivas para esses pacientes, onde contam com atividades sócioeducativas direcionadas para o enfrentamento da doença ${ }^{23}$. No Brasil não há relatos dessas práticas e os programas de saúde do governo não investem em educação para as doenças neuromusculares; suas ações ficam reduzidas à concessão dos ventiladores de suporte à vida e a medicamentos que apresentam alguma eficácia ${ }^{24}$.

A estratégia de criar um jogo eletrônico para explicar a Distrofia Muscular de Duchenne mostrou-se didaticamente interessante, pois a análise pré e pós-teste resultou no aumento de repertório, exceto para o tema "órteses" em pacientes deambuladores - esse fato pode ser relacionado à fase da doença em que eles se encontram, na qual o uso de órteses para membros inferiores é extremamente estimulado pela equipe de reabilitação, por prevenir evolução de encurtamentos ${ }^{25}$ e, dessa forma, prolongar o tempo de capacidade de marcha.

A análise do discurso qualitativo ${ }^{26}$ dos pacientes em relação ao jogo mostrou satisfação e interesse por eles, e pode ser uma ferramenta para essa população divulgar e quebrar paradigmas sobre a doença.

Todo o processo de criação do jogo eletrônico Duchsville foi realizado pela equipe transdisciplinar, inclusive todos os ajustes de execução foram baseados nas opiniões dos pacientes testados, aproximando estudantes de Engenharia e Design dos profissionais da saúde e da população-alvo.

\section{CONCLUSÃO}

O jogo eletrônico interativo DuchsVille foi criado, avaliado e pode ser utilizado como uma ferramenta 
Klein NA, et al. Desenvolvimento e avaliação de jogo eletrônico interativo. Rev Ter Ocup Univ São Paulo. 2015 maio/ago.;26(2):281-7.

de tecnologia de informação aplicável para favorecer processos de ensino e aprendizagem sobre aspectos de reabilitação na doença Distrofia Muscular de Duchenne,

\section{REFERÊNCIAS}

1. Kakulas BA. Problems and solutions in the rehabilitation of patients with progressive muscular dystrophy. Scand J Rehab Med Suppl. 1999;39:23-37. doi: 10.1080/003655098443869.

2. Manzur AY, Kinali M, Mutoni F. Up date on the management of Duchenne muscular dystrophy. Arch Dis Child. 2008;93:986-90. doi: 10.1136/adc.2007.118141.

3. Liu M, Mineo K, Hanayama K, Fujiwara T, Chino N. Practical problems and management of seating through the clinical stages of Duchenne's muscular dystrophy. Arch Phys Med Rehabil. 2003;84:818-24. doi: 10.1016/S00039993(02)04953-5.

4. Merlini L, Cicognani A, Malaspina E. Early prednisone treatment in Duchenne muscular dystrophy. Muscle Nerve. 2003;27:222-7. DOI: 10.1002/mus.10319.

5. Floriani CA. Home-based palliative care: challenges in the care of technology-dependent children. J Pediatr (Rio J). 2010;86(1):15-20. DOI: http://dx.doi.org/10.1590/S002175572010000100004.

6. Nereo N, Fee R, Hinton V. Parental stress in mothers of boys with Duchenne muscular dystrophy. J Pediatr Psychol. 2003;28:473-84. DOI: 10.1093/jpepsy/jsg038.

7. Anderson JL, Head SI, Rac C, Morley JW. Brain function in Duchenne muscular dystrophy. Brain. 2002;125:4-13. doi: http://dx.doi.org/10.1093/brain/awf012.

8. Mehler MF. Brain dystrophin, neurogenetics and mental retardation. Brain Res Rev. 2000;32:277-307. doi: 10.1016/ S0165-0173(99)00090-9.

9. Stone K, Tester C, Blakeney J, Howarth A, McAndrew H, et al. Occupational therapy and Duchenne muscular dystrophy. Chichester, England: John Wiley \& Sons; 2007.

10. Webb CL. Parents' perspectives on coping with Duchenne muscular dystrophy. Child Care Health Dev. 2005;31(4):38589. doi: 10.1111/j.1365-2214.2005.00518.x.

11. Nielsen J, Tahir M. Homepage usabilidade: 50 Websites. Desconstruídos. São Paulo: Elsevier; 2002.

12. Valente JA. Por que o computador na educação. Campinas: Gráfica Central da Unicamp; 1993. p.24-44.

13. Campos FCA, Rocha ARC. Design instrucional e construtivismo: em busca de modelos para o desenvolvimento de Software. In: Anais do IV Congresso aproximando o diálogo da lógica técnico-científica dos profissionais da saúde da lógica do senso comum da população. de Rede Iberoamericana de Informática Educativa (RBIE). Brasília; 1998. Disponível em: http://www.ufrgs.br/niee/ eventos/RIBIE/1998/pdf/com_pos_dem/250M.pdf.

14. Moretti FA, Heymann RE, Marvulle V, Pollak DF, Rieira R. Avaliação do nível de conhecimento sobre fibromialgia entre usuários da internet. Rev Bras Reumatol. 2011;51(1):13-9. doi: http://dx.doi.org/10.1590/S0482-50042011000100002.

15. Wetstone SL, Sheehan TJ, Votanw RG. Evaluation of a computer based education lesson for patients with rheumatoid arthrit. J Rheumatol. 1985;12:(5):907-12.

16. Henriksson C, Carlberg U, Kjällman M, Lundberg G, Henriksson K.G. Evaluation of four outpatient educational programmes for patients with longstanding fibromyalgia. J Rehabil Med. 2004;36(5):211-9. doi: 10,1080/16501970410027494.

17. Klein NA, Oliveira ASB, Nakazune SJ, Takizawa MM, Arai JS. MIBRELA orientation Brazilian software for amyotrophic lateral sclerosis patients. Rev Neurocienc. 2007;15(1):44-8. Available from: http:// www.ipg.org.br/ipg/project/ipg/public/uploads/site/ downloads/0961d6132a2d2a840b71ca8a0b105d6d.pdf.

18. Curran K, George C. The future of web and mobile game development. Int J Cloud Comput Serv Sci. 2012;1(1):2534. Available from: http://iaesjournal.com/online/index. php/IJ-CLOSER/article/view/Londonderry\%2C\%20UK/ pdf

19. Magalhães CR. O jogo como pretexto educativo: educar e educar-se em curso de formação em saúde. Interface Comunic Saúde Educ (Botucatu). 2007;11(23):647-54. doi: http://dx.doi.org/10.1590/S1414-32832007000300021.

20. Toscani NV, Santos AJ, Silva LM, Tonial CT, Mezzari A. Desenvolvimento e análise de jogo educativo para crianças visando à prevenção de doenças parasitológicas. Interface Comunic Saúde Educ (Botucatu). 2007;11(22):281-294. doi: http://dx.doi.org/10.1590/S1414-32832007000200008.

21. Bittencourt JR, Giraffa LM. Modelando ambientes de aprendizagem virtuais utilizando role-playing games. In: Anais do XIV Simpósio Brasileiro de Informática na Educação (SBIE). Rio de Janeiro: NCE - IM/UFRJ; 2003. Disponível em: http://www.nce.ufrj.br/sbie2003/ publicacoes/paper71.pdf. 
Klein NA, et al. Desenvolvimento e avaliação de jogo eletrônico interativo. Rev Ter Ocup Univ São Paulo. 2015 maio/ago.;26(2):281-7.

22. MDA Fighting Muscle Disease. MDA Summer Camp is special [cited 2013 Dec 12]. Available from: http://mda.org/ summer-camp.

23. Ishikawa Y. Manual for the care of patients using noninvasive ventilation. Matsudo, Japan: Japan Planning Center Inc.; 2005.

24. Gomide MH, Rose N, Langer AL. ABDIM - Associação Brasileira de Distrofia Muscular. Conheça seus direitos. São Paulo: ABDIM; 2012 [citado 09 jan. 2014]. Disponível em: http://www.abdim.org.br/wp-content/uploads/2012/10/ cartilha-word-docx-out-12-PDF-ok.pdf.

25. Bushby K, Bourke J, Bullock R, Eagle M, Gibson M, Quinby J. The multidisciplinary management of Duchenne muscular dystrophy. Curr Pediatr. 2005;15:292-300. doi: 10.1016/j.cupe.2005.04.001.

26. Minayo MCS. O desafio do conhecimento - pesquisa qualitativa em saúde. 8a ed. São Paulo: Hucitec; 2004.

Recebido para publicação: 21.03.14

Aceito para publicação: 06.05.15 